\title{
Types of gas fluidization of cohesive granular materials
}

\author{
Jose Manuel Valverde and Antonio Castellanos \\ Faculty of Physics, Universidad de Sevilla, Avenida Reina Mercedes s/n, 41012 Sevilla, Spain \\ (Received 22 May 2006; revised manuscript received 3 January 2007; published 26 March 2007)
}

\begin{abstract}
Some years ago it was shown that gas-fluidized powders may transit from solidlike to fluidlike fluidization prior to bubbling, shedding light on a long-standing controversy on the nature of "homogeneous" fluidization. In this paper it is shown that some gas-fluidized powders may also transit from the fluidlike regime to elutriation, with full suppression of the bubbling regime. We provide a diagram that can be used to predict these types of fluidization exhibited by cohesive powders based on simple phenomenological equations in which particle aggregation due to attractive forces is a key ingredient.
\end{abstract}

DOI: 10.1103/PhysRevE.75.031306

PACS number(s): 45.70. $-\mathrm{n}$, 81.05.Rm, 47.55.Lm, 47.57.Gc

Processing and handling of granular materials in fluidized beds is widespread in industry because of their multiple advantages such as enhanced fluid-solid contact and improved flowability. In a typical fluidized bed the material rests on a porous plate through which fluid is pumped to the granular bed. Granular beds fluidized by gas are, however, usually unstable, most of the fluid bypassing the bed through large bubbles which curtail uniform expansion and hamper the quality of fluid-solid mixing. The relative importance of interparticle forces and hydrodynamic forces in the distinction between nonbubbling and bubbling fluidization is still under debate [1-3]. A relevant parameter that characterizes the cohesiveness of a granular material is the granular Bond number Bo, defined as the ratio of interparticle attractive force to particle weight. The attractive force $F_{0}$ between uncharged particles fluidized by dry gas arises mainly from the van der Waals interaction, $F_{0} \simeq A d_{a} / 24 z_{0}^{2}$, where $z_{0} \simeq 3-4 \AA$ is the distance of closest approach between two molecules, $A$ is the Hamaker constant, and $d_{a}$ is the typical size of the surface asperities [4]. Typically $A \sim 10^{-19} \mathrm{~J}$ and $d_{a} \sim 0.2 \mu \mathrm{m}$ [4], which gives an estimate of $F_{0} \sim 10 \mathrm{nN}$. In the case of noncohesive particles $\left(\mathrm{Bo} \lesssim 1\right.$, particle size $\left.d_{p} \gtrsim 100 \mu \mathrm{m}\right)$ it is generally seen that gas-fluidized beds bubble just beyond the minimum fluidization point (Geldart $B$, bubbling powders $[5])$. For slightly cohesive beads $(\mathrm{Bo} \gtrsim 1)$, gas-fluidized beds exhibit a uniform fluidization interval of short extension, characterized by a stable expansion and solidlike behavior. For these beds the fluidlike regime initiates just at the bubbling onset (Geldart $A$, aeratable powders [5]).

Traditionally, it has been believed that cohesive particles (Bo $\gg 1$, typically $d_{p} \lesssim 20 \mu \mathrm{m}$ ) are impossible to fluidize uniformly by gas due to crack formations and channeling favored by strong cohesive forces (Geldart $C$, cohesive powders [5]). The Geldart diagram [5], which was originally based on empirical observations on beds fluidized by air at ambient conditions, has been of great help for many years to predict the type of gas-fluidization expected for a granular material. However, the Geldart diagram, based on particle size and density, is not currently a useful tool to predict the fluidization behavior of many fine cohesive powders. There is an increasing number of recent reports revealing nonbubbling fluidlike fluidization for some fine powders in contrast with the predicted Geldart $C$ behavior. Preconditioning methods (surface coating, magnetic assistance, vibration, ultra- sonic excitation, etc.) have been developed that help to erase powder memory on previous process allowing for nonbubbling fluidlike fluidization [6-8]. For example, powders of micrometer-sized particles, with cohesion reduced by addition of surface additives, have been shown to transit from the solidlike regime to a nonbubbling fluidlike one, and then to bubbling at higher gas velocities [6]. Similarly powders of dense nanoparticles (such as titania) have been shown to exhibit a transition to bubbling at high gas velocities, whereas powders of moderate density nanoparticles (such as silica) can be uniformly fluidized and experience a transition to elutriation with full suppression of bubbles [8]. Moreover, it has been seen that a change of gas viscosity has an important effect on the fluidlike regime, enhancing bed expansion, and delaying the onset of bubbling [9]. Clearly these types of fluidization cannot be predicted by the original Geldart diagram. Thus there is a need for an extended diagram that is able to predict the transitions between the different types of fluidization behavior and the influence of physical parameters such as particle size and density but also interparticle force and gas viscosity, which play a relevant role according to experimental observations.

Previous works have shown a similarity of behavior, at both macroscopic and mesoscopic scales, between liquidfluidized beds of noncohesive beads and gas-fluidized beds of preconditioned fine cohesive powders [10]. In this work we use empirical relationships well known for liquid fluidization of noncohesive beads and modify it to take into account aggregation in gas fluidization of cohesive particles. This simple approach allows us to map the fluidization behavior boundaries of preconditioned cohesive powders. We show that the new types of gas-fluidization behavior observed for micro- and nanopowders can be predicted from this extended diagram. The effect of gas viscosity is specially addressed and additional experimental results are presented to illustrate the validity of the proposed diagram.

Harrison et al. [11] hypothesized that fluid bubbles in the fluidlike regime are no longer stable if their rising velocity $U_{b}$ exceeds the terminal settling velocity of the individual particles $v_{p 0} \simeq(1 / 18)\left(\rho_{p}-\rho_{f}\right) d_{p}^{2} g / \mu$ in the laminar regime, where $\rho_{p}$ is the particle density, $\rho_{f}$ is the fluid density, $\mu$ is the fluid viscosity, and $g$ is the gravitational acceleration. Using the semiempirical relation $U_{b} \simeq 0.7 \sqrt{g D}$, where $D$ is the bubble size, and neglecting fluid inertia we arrive then at 
$D_{m} / d_{p} \simeq\left(\rho_{p}-\rho_{f}\right)^{2} g d_{p}^{3} /(13 \mu)^{2}$ for the largest stable size of the bubbles, $D_{m}$. Harrison et al. carried out an extensive series of experimental observations mostly on liquid-fluidized beds of noncohesive beads with varying $\mu, \rho_{p}$, and $\rho_{f}$ and concluded that the ratio $D_{m} / d_{p}$ coincided with bubbling behavior when $D_{m} / d_{p} \gtrless 10$, and with uniform fluidization when $D_{m} / d_{p} \lesssim 1$. The regime of behavior described approximately by 1 $\leqslant D_{m} / d_{p} \leqslant 10$ defined a transition from nonbubbling to bubbling behavior. For example, lead shot particles could be uniformly fluidized by a 75 wt $\%$ aqueous solution of glycerol $\left(D_{\max } / d_{p}=1.9\right)$, but experienced bubbling using pure water $\left(D_{\max } / d_{p}=14.8\right)$. Interestingly Harrison et al. reported on beds of light phenolic microballoons $\left(\rho_{p} \simeq 0.24 \mathrm{~g} / \mathrm{cm}^{3}\right.$, $d_{p} \simeq 125 \mu \mathrm{m}$ ) fluidized with $\mathrm{CO}_{2}$ at various pressures, showing bubbling behavior at ambient pressure $\left(D_{m} / d_{p} \simeq 24\right)$ but nonbubbling fluidlike behavior at high gas pressures $\left(D_{m} / d_{p} \simeq 2\right)$. This finding contradicts the common misconception that gas-fluidized beds of noncohesive particles do always bubble in the fluidlike regime.

On the other hand, following Wallis's ideas, some authors [12] have suggested that stable bubbles in fluidized beds are an outcome of the formation of concentration shocks in particle concentration when the propagation velocity of a voidage disturbance $u_{\phi}$ surpasses the elastic wave velocity $u_{e}$ of the bed. It is well accepted that $u_{\phi}$ can be derived from the relation $u_{\phi}=-\phi d v_{g} / d \phi$, where the gas velocity $v_{g}$ is related to the particle volume fraction $\phi$ by the Richardson-Zaki equation $v_{g}=v_{p 0}(1-\phi)^{n}, n \simeq 5.6$ being in the small Reynolds number limit [13]. The elastic wave velocity $u_{e}$ is given by $u_{e}=\left[\left(1 / \rho_{p}\right)(\partial p / \partial \phi)\right]^{1 / 2}$ where $p$ is the particle phase pressure endowed by particle velocity fluctuations. There is not a general consensus on a correct formulation of $p(\phi)$ as recently discussed by Guazzelli [14]. It is clear that $p$ should increase with $\phi$ at low volume fractions, reach a maximum, and then decrease for larger $\phi$ [2], but a reliable equation for $p$ is completely unknown. In our work we will use the equation $p \sim \rho_{p} g d_{p} \phi^{2}$, which is upheld by measurements of the collisional pressure in a water-fluidized bed [15], by recent numerical studies [14], and by the theoretical work of Batchelor in the dilute limit [12]. A similar relationship was also derived by Rapagna et al. [16]. Batchelor [12] and Jackson [1] pointed out, however, that this derivation was conceptually wrong, albeit Rapagna et al. found good agreement with observations on the initiation of visible bubbling under systematic variations of relevant parameters such as pressure, temperature, and addition of fines [16]. Zenit et al. [15] measured the collisional pressure in beads of glass, plastic, and steel particles of various sizes fluidized by water. Their experimental results were shown to agree with the quadratic dependence used by Rapagna et al., whereas results of collision-based models, suggesting a linear dependence on $\phi$, were in poor correspondence with the experiments. Fluidized beds were also extensively studied by Batchelor [12], who formulated a theory to predict the onset of bubbling. In his theoretical work, Batchelor recognized that a reliable equation for particle pressure in a fluidized bed is completely unknown. He wrote $p=\rho_{p} \phi H v_{g}^{2}$, were $v_{g}$ is the gas velocity, and for $H(\phi)$ he used the simple assumption $H=\left(\phi / \phi_{J}\right)(1$ $-\phi / \phi_{J}$ ), which gave a quadratic dependence of $p$ on $\phi$ in the dilute limit. Batchelor concluded his paper [12] saying that "we may note that the specific criterion for instability found by Foscolo and Gibilaro and compared with numerous observations happens to be not very different numerically from the criterion found herein." We believe therefore that the functional dependence $p \sim \rho_{p} g d_{p} \phi^{2}$ can be at least justified from the empirical point of view.

Cohesive particles aggregate in a gas-fluidized bed driven by the dynamic equilibrium between interparticle attractive force $F_{0}$ and flow shear, which supports the particle weight in the gravity field. Assuming that the maximum shear force that the aggregate in the fluidized bed can stand is of order of $F_{0}$, the scaling law Bo $\sim N k^{2}=k^{D+2}$ was derived to estimate the aggregate size $d^{*}$ [3]. Here $N$ is the number of particles aggregated, $k$ is the ratio of $d^{*}$ to particle size $d_{p}$, and $D$ $=\ln N / \ln k$. It is clear that Harrison's and Wallis's criteria are not suitable for fluidized beds of aggregated particles. The simplest approach to account for aggregation is to consider aggregates as effective particles of size $d^{*}$ and reduced density $\rho^{*}=\rho_{p} N / k^{3}$. (It can be interesting to establish an analogy between our light aggregates and the light phenolic microballoons uniformly fluidized by Harrison et al. by gas [11].) As reported in previous work [17] this approach is useful to describe fluidized bed expansion and settling by means of a modified Richardson-Zaki law, $v_{g}=v^{*}\left(1-\phi^{*}\right)^{n}$, where $v^{*}=v_{p 0} N / k$ is the terminal settling velocity of a single aggregate and $\phi^{*}=\phi k^{3} / N$ is the volume fraction of the aggregates in the fluidized bed. Analogously, the Harrison's modified equation that results from the balance between $U_{b}$ and the settling velocity of a single aggregate $v^{*}$ is

$$
\frac{D_{m}}{d^{*}} \simeq \frac{1}{18^{2} 0.7^{2}} \frac{\rho_{p}^{2} g d_{p}^{3}}{\mu^{2}} \mathrm{Bo}^{(2 D-3) /(D+2)}
$$

and, using the modified Richardson-Zaki equation, we can write the modified Wallis criterion as

$$
\begin{gathered}
u_{\phi}^{*} \simeq \phi \frac{1}{18} \frac{\rho_{p} g d_{p}^{2}}{\mu} n\left(1-\phi \mathrm{Bo}^{(3-D) /(D+2)}\right)^{n-1} \mathrm{Bo}^{2 /(D+2)}, \\
u_{e}^{*} \simeq\left(g d_{p} \phi \mathrm{Bo}^{(4-D) /(D+2)}\right)^{1 / 2}, \\
u_{\phi}^{*}=u_{e}^{*} \text { at bubbling onset. }
\end{gathered}
$$

On the other side, at small gas velocities the fluidlike nonbubbling regime is limited by jamming of the fluidized aggregates. At the fluid-to-solid transition these aggregates jam in a solidlike state with a particle volume fraction $\phi_{J}$ $=\phi_{J}^{*} k^{D-3} \approx \phi_{J}^{*} \mathrm{Bo}^{(D-3) /(D+2)}$, where $\phi_{J}^{*}$ is the volume fraction of the jammed aggregates [18]. Thus the bed will transit through the solidlike fluidization regime when $\phi<\phi_{J}$.

Let us denote by $\phi_{b}$ a solution of Eq. (2) in case it exists and $\phi_{s}>\phi_{J}$ the particle volume fraction of the powder bed in its initial settled state. We may have then two possibilities.

(1) If $\phi_{b} \geq \phi_{s}$, it is expected that the fluidized bed transits from the initial settled state to the bubbling regime without further expansion as soon as the gas velocity $v_{g}$ surpasses the minimum fluidization velocity (Geldart $B$ behavior).

(2) If $\phi_{b}<\phi_{s}$, the system will exhibit an expanded nonbubbling fluidization regime. For $\phi_{b}<\phi_{J}$, the bed might 


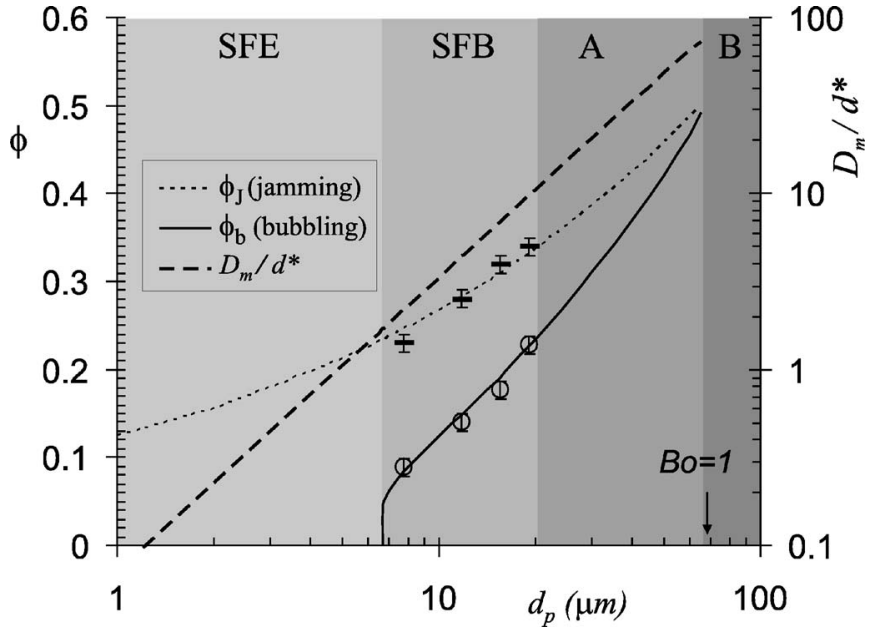

FIG. 1. Phase diagram determining the transition between the types of fluidization behavior as a function of particle size. Left axis: particle volume fraction at the jamming transition $\phi_{J}$ and at the transition to bubbling $\phi_{b}$. Right axis: Ratio of the maximum stable size of a fluid pocket to particle size in the fluidlike regime. $\rho_{p}=1135 \mathrm{~kg} / \mathrm{m}^{3}, \rho_{f}=1 \mathrm{~kg} / \mathrm{m}^{3}, \mu=1.79 \times 10^{-5} \mathrm{~Pa} \mathrm{~s}, F_{0}=2 \mathrm{nN}, g$ $=9.81 \mathrm{~m} / \mathrm{s}^{2}, \phi_{J}^{*}=0.51$, and $D=2.5$. Experimental data on the jamming and bubbling transitions for fluidized beds of Xerox toners [3] are shown.

even show a solidlike regime followed by fluidlike regime and a transition to bubbling at high gas velocities [solidliketo-fluidlike-to-bubbling (SFB) behavior]. The existence of a nonbubbling fluidlike regime depends, however, on the necessary condition $D_{m} / d^{*} \lesssim 10$. Otherwise the stable fluid pockets can reach a macroscopic size leading to bubbling behavior just above the jamming transition. In the latter case we would have a transition from expanded solidlike to bubbling regime (Geldart $A$ behavior).

Using Eq. (1) in Eq. (2) it can be shown that $u_{e}^{*}-u_{\phi}^{*}$ $=u_{e}^{*}\left[1-0.7 n\left(D_{m} / d^{*}\right)^{1 / 2}\left(\phi^{*}\right)^{1 / 2}\left(1-\phi^{*}\right)^{n-1}\right]$. Since $\left(\phi^{*}\right)^{1 / 2}(1$ $\left.-\phi^{*}\right)^{n-1}$ takes a maximum value of 0.195 at $\phi^{*}=0.098$, we have that $\min \left(u_{e}^{*}-u_{\phi}^{*}\right)>0$ for $D_{m} / d^{*}<1.72$. Thus for $D_{m} / d^{*}<1.72$ it is $u_{e}^{*}>u_{\phi}^{*} \forall \phi>0$, indicating the absence of a transition to bubbling. Therefore both criteria coincide in predicting that the system will transit from the solidlike regime to a nonbubbling fluidlike regime and from the fluidlike regime to elutriation (SFE behavior) with full suppression of bubbling for $D_{m} / d^{*}<1.72$. To our knowledge this is the first time that a direct correlation between Harrison's and Wallis's criteria is analytically established.

In Fig. 1 we plot $\phi_{b}, \phi_{J}$, and $D_{m} / d^{*}$ as a function of particle size $d_{p}$. We have used $\rho_{p}=1135 \mathrm{~kg} / \mathrm{m}^{3}, \mu=1.79$ $\times 10^{-5} \mathrm{~Pa} \mathrm{~s}, F_{0}=2 \mathrm{nN}, \phi_{J}^{*}=0.51$, and $D=2.5$. These values correspond to fluidization of xerographic toners (polymer particles coated with silica nanoparticles) by dry nitrogen at ambient conditions of pressure and temperature [3]. Surface coating with silica nanoparticles decreases the typical surface asperity size down to the size of nanoparticle aggregates that are dispersed on the particle surface (of size around $50 \mathrm{~nm}$ [3]), and thus decreases $F_{0}$ [3]. Data for $\phi_{J}$ and $\phi_{b}$ from experiments on xerographic toners previously reported (see Ref. [3] for experimental details) have been plotted, showing good agreement with the expected values.
From Fig. 1 we predict Geldart $B$ behavior for $d_{p}$ $>70 \mu \mathrm{m}$, Geldart $A$ behavior for $20<d_{p}<70 \mu \mathrm{m}$, SFB behavior for $6.7<d_{p}<20 \mu \mathrm{m}$, and SFE behavior for $d_{p}$ $<6.7 \mu \mathrm{m}$. Remarkably the $A-B$ boundary coincides with $\mathrm{Bo} \simeq 1$, which is also the limiting condition for aggregation $(k>1)$. The criterion $\mathrm{Bo} \simeq 1$ for the $A-B$ boundary was already derived by Molerus [19] from analysis of experimental data and by Rhodes et al. [20] using discrete element modeling. However, the $A-B$ boundary cannot be sharply defined since the existence of nonbubbling expansion beyond minimum fluidization for slightly cohesive powders is difficult to observe and moreover depends on the history of the sample. For noncohesive hard spheres settled in the gravity field the particle volume fraction of the settled bed, $\phi_{s}$, only varies slightly between 0.6 (random loose packing) and 0.64 (random close packing). Thus near $\mathrm{Bo} \simeq 1$ bed expansion is hard to measure and usually depends on experimental details, such as for example heterogeneous distribution of gas flow due to nonuniform porous distributor plates [21]. This is neatly illustrated in the work by Sundaresan [2], who observed the fluidization behavior of glass beads $\left(\rho_{p}\right.$ $\simeq 2500 \mathrm{~kg} / \mathrm{m}^{3}$ ) in the size range $60 \lesssim d_{p} \lesssim 200 \mu \mathrm{m}$. The author observed that for $d_{p} \lesssim 100 \mu \mathrm{m}$ the bed maintained a solidlike smooth appearance with no sustained bubbling (Geldart $A$ behavior). For $d_{p} \gtrsim 100 \mu \mathrm{m}$ the window of stable expansion was very small, which was consistent with Geldart $B$ behavior. However, occasional bubbles could be observed near the top free surface for $88 \mu \mathrm{m}$ sized beads when the gas velocity was only slightly larger than the minimum fluidization velocity (Sundaresan defined the bubbling condition when bubbles were observed at a rate of about one bubble per minute). Interestingly the limit $\mathrm{Bo} \simeq 1$ for this material (using $A \simeq 1.5 \times 10^{-19} \mathrm{~J}$ for glass [22] and $d_{a}=0.2 \mu \mathrm{m}$ ) is found for $d_{p} \simeq 90 \mu \mathrm{m}$.

We must note that the parameters used to plot the type of diagram in Fig. 1 depend on the particular conditions of each system. For example, the mechanism of aggregation could have an influence in the type of fluidization behavior. Experimental measurements indicate that $D=2.5$ for aggregates of fine particles, according to the diffusion-limited particle-tocluster aggregation (DLA) model. DLA seems to be the dominant mechanism of aggregation in fluidized beds of fine particles. Even in the case of nanoparticles, experiments indicate that preexisting simple aggregates aggregate into complex aggregates according to the DLA mechanism [23,24]. This is in contrast with other aggregation models, such as diffusion-limited cluster-to-cluster aggregation, which give lower values of the fractal dimension $(D=1.8)$. Although we have used $D=2.5$ to illustrate our work, the general equations could be used to study the effect of the mechanism of aggregation as $D$ changes. The size of the aggregates decreases as particle size increases. When particles are just slightly cohesive, the use of the term fractal dimension does not have a physical justification since $D=\ln N / \ln k$ may show a large variation when aggregates are formed by just a few particles. However, in this limiting case, in which Bo is close to 1 , the value of $D$ does not have a remarkable effect on the results and the original criteria for nonaggregated particles give results close to the results obtained using the modified equations for cohesive aggregated particles. Only 
in the case of fine particles does our modified criterion really make a difference and provide a relevant contribution. Of course, in the cases where $\mathrm{Bo}<1$ for which cohesive aggregation is not expected we must use the empirical equations for individual nonaggregated particles.

The value of $\phi_{J}^{*}=0.51$ used in the plot of Fig. 1 is the volume fraction of aggregates at the jamming threshold measured experimentally for xerographic toners with varying particle size in the range from 7.8 to $19.1 \mu \mathrm{m}$ and $32 \%$ of surface additive coverage (SAC) [18]. The condition $\mathrm{Bo}=1$ (limit of no aggregation) is found for $d_{p}=70 \mu \mathrm{m}$ and using the Wallis criterion we obtain $\phi_{b}=0.51$ at the transition to bubbling for $d_{p}=70 \mu \mathrm{m}$. If we assume that jamming of $70 \mu \mathrm{m}$ sized nonaggregated particles occurs at $\phi_{J}=0.51 \mathrm{we}$ conclude that there cannot be an interval of nonbubbling fluidlike expansion for $d_{p}>70 \mu \mathrm{m}$. The interesting point is that the volume fraction of aggregates of fine particles at jamming coincides with the volume fraction at the transition to bubbling in the limit $\mathrm{Bo}=1$, which is the limit identified by other authors as the Geldart $A-B$ boundary. We have also made measurements on toners with higher additive coverage and we have obtained that the volume fraction of the agglomerates at the jamming transition increases as the surface coverage of additive is increased. For example, we measured $\phi_{J}^{*} \simeq 0.56$ for $64 \%$ SAC [18].

If we use in our calculations a different set of physical parameters, such as for example $\rho_{p}=2500 \mathrm{~kg} \mathrm{~m}^{3}$ and $F_{0}$ $=8 \mathrm{nN}$ (typically corresponding to glass beads, $A=1.5$ $\times 10^{-19} \mathrm{~J}, d_{a} \simeq 0.2 \mu \mathrm{m}$ ) the limit $\mathrm{Bo}=1$ is found for $d_{p}$ $=85 \mu \mathrm{m}$, which gives $\phi_{b}=0.62$ at the bubbling transition, in agreement with observations by Sundaresan on the $A-B$ boundary [2]. Since a value of $\phi=0.62$ is close to the random close packing limit, we would conclude that settled beds of $d_{p} \geqslant 85 \mu \mathrm{m}$ glass beads would exhibit inappreciable expansion at the bubbling transition as is seen experimentally. For smaller particles $(\mathrm{Bo}>1)$ we would use $\phi_{J}^{*}=0.62$ to determine $\phi_{J}$. Note that the value of $\phi_{J}^{*}$ is only used in our paper to identify the $A-B$ boundary and does not play a role in either Harrison's or Wallis's modified criteria to predict the fluidization behavior of fine particles, which is the main goal of our work.

In the modified Wallis criterion we have used a model for particle pressure with a quadratic dependence on particle volume fraction, which is a subject of controversy as pointed out earlier. Using this quadratic dependence the predicted results on the particle volume fraction at the bubbling transition agrees with the experimental results available (Fig. 1). If we had used instead a linear dependence, full suppression of bubbling (SFE behavior) would have been predicted for particle sizes smaller than $25 \mu \mathrm{m}$, against the experimental evidence (we observe a transition to bubbling for 7.8, 11.8, 15.4 , and $19.1 \mu \mathrm{m}$ particle sizes). Moreover, if we had used a linear dependence, SFE behavior $\left[\min \left(u_{e}^{*}-u_{\phi}^{*}\right)>0\right]$ would have been predicted in general for $D_{b} / d^{*}=12.5$, which is against the extensive observations on liquid-fluidized beds made by Harrison et al. [11], suggesting the condition $D_{b} / d^{*} \lesssim 1$ for full suppression of macroscopic bubbling.

Figure 2(a) shows the effect of particle density $\rho_{p}$ on the gas-fluidization behavior. This is the type of diagram originally presented by Geldart [5] to distinguish between the
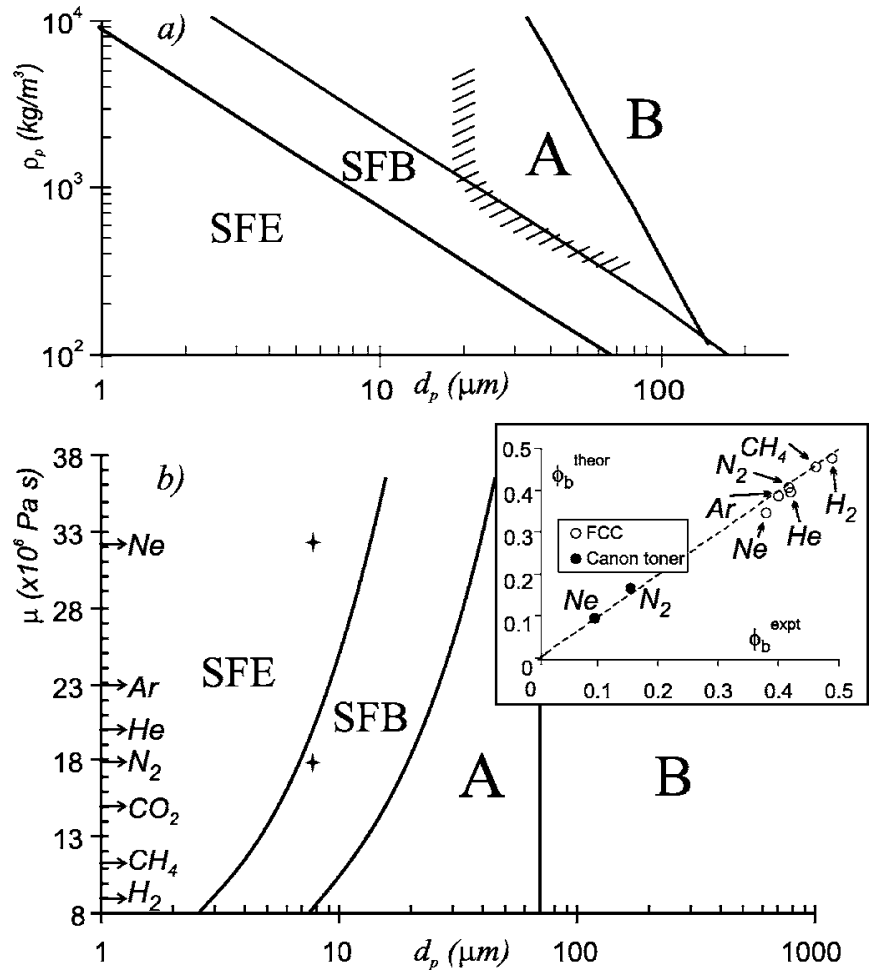

FIG. 2. Effect of particle density (a) and gas viscosity (b) on the boundaries between types of fluidization shown in Fig. 1. The shaded line represents the boundary between $A$ and $C$ powders as shown in the original Geldart diagram (Ref. [5]). The inset of (b) shows the particle volume fraction theoretically derived vs experimentally measured at bubbling onset for two powders. Fresh cracking catalyst (FCC), showing $A$ behavior, and Canon toner, showing SFB behavior, are fluidized with different gases (indicated) at ambient conditions.

different types of fluidization behavior. In this plot we have delineated also the boundary drawn by Geldart [5] to separate empirically cohesive $C$ powders and aeratable $A$ powders. Interestingly the SFB- $A$ boundary matches the Geldart $C-A$ boundary for moderate values of particle density. It must be remarked that the original Geldart diagram was derived from experiments on history-dependent samples, typically cohesive powders for which fluidization was not preceded by a preconditioned procedure. Interparticle adhesive forces are largely increased by stresses applied during powder history [3] and, as a consequence, large coherent fragments of the consolidated powder are difficult to break by the gas, giving raise the classical Geldart $C$ behavior characterized by strongly heterogeneous fluidization (rising plugs, rat holes, channeling, etc.). This memory effect is minimized by coating the particles with surface additives. Fluidization can also be made possible by vibration, placing magnetic beads within the powder that are agitated by an oscillating magnetic field, centrifugation or by means of sound wave excitation at low frequencies [7]. Convenient preconditioning facilitates fluidization by breaking contacts down to the level of primary particles, thus erasing powder memory and allowing individual particles to aggregate in fractal agglomerates. Our diagram is able to predict which type of fluidization is to 
be expected, either SFB or SFE, for preconditioned fine cohesive powders.

Recent studies have demonstrated that uniform gasfluidization allows for the massive area of nanoparticles to be utilized for gas-solid catalytic reaction engineering $[8,25]$. When silica nanoparticles $\left(d_{p}=12 \mathrm{~nm}, \rho_{p}=2560 \mathrm{~kg} / \mathrm{m}^{3}\right)$ are fluidized by nitrogen, SFE behavior is experimentally observed [8], whereas SFB behavior is reported for fluidization of titania nanoparticles $\left(d_{p}=21 \mathrm{~nm}, \rho_{p}=4500 \mathrm{~kg} / \mathrm{m}^{3}\right)$. A further extension of our diagram would enable us to predict the type of fluidization expected for preconditioned nanopowders. The study of nanopowders requires, however, additional elaboration since for these powders the effective primary particles in fluidization are simple agglomerates that already exist prior to fluidization [23]. The typical density and size of these simple agglomerates for silica nanopowder are $\rho_{s} \approx 50 \mathrm{~kg} / \mathrm{m}^{3}$ and $d_{s} \approx 30 \mu \mathrm{m}[8,23]$, which according to Fig. 2(a) would give SFE behavior in agreement with experimental observations. For titania nanopowders simple agglomerates are denser $\left(\rho_{s} \approx 100 \mathrm{~kg} / \mathrm{m}^{3}\right)$ [8], which shifts the fluidization behavior to SFB as seen experimentally [8].

The effect of fluid viscosity on the boundaries between the different types of fluidization behavior is shown in Fig. 2(b). In accordance with the diagram, it has been observed that Geldart $A$ powders at ambient conditions can be uniformly fluidized with substantial expansion under conditions at elevated temperature [16]. Likewise improvement of fluidization quality and enhanced fluidlike expansion have been reported when high viscosity gases are used in gas fluidization of Geldart $A$ powders [4]. The inset of Fig. 2(b) shows good agreement between the values of the particle volume fraction at the transition to bubbling $\phi_{b}$ theoretical and the experimental for two different cohesive powders fluidized with different gases, Geldart $A$ FCC catalyst $\left(\rho_{p}\right.$ $\left.\simeq 887 \mathrm{~kg} / \mathrm{m}^{3}, d_{p} \simeq 59.4 \mu \mathrm{m}\right)$ [4], and Canon CLC700 toner $\left(\rho_{p} \simeq 1200 \mathrm{~kg} / \mathrm{m}^{3}, d_{p} \simeq 8.5 \mu \mathrm{m}, F_{0} \simeq 10 \mathrm{nN}\right)$ exhibiting SFB behavior (see Ref. [3] for experimental details).

Figure 2(b) predicts that a $7.8 \mu \mathrm{m}$ particle sized Xerox toner $\left(\rho_{p} \simeq 1135 \mathrm{~kg} / \mathrm{m}^{3}, F_{0} \simeq 2 \mathrm{nN}\right)$ showing SFB behavior when fluidized with nitrogen (as seen in Fig. 1) would shift its behavior to SFE when fluidized with neon [see symbols in main graph of Fig. 2(b)]. To account for this change of behavior we have carried out further experimental measurements. Our fluidized bed consists of a vertical 4.42-cm-diam vessel containing the powder and mounted on a electromagnetic shaker. The bed is subjected to a controlled flow of gas injected through a sintered metal gas distributor at its bottom. We use nitrogen and neon as fluidizing gases at ambient conditions. In all the measurements the powder bed is initialized by subjecting it to a high gas flow coupled to strong shaking. In this initial state the powder loses memory of previous processes [26]. Then the gas flow is decreased to the desired value and measurements are taken once the system has reached a reproducible stationary state. The particle volume fraction of the fluidized bed $\phi$ is derived from the height of the bed, which is measured by an ultrasonic sensor placed on top of the vessel. This device can determine distance with an accuracy of $\pm 0.01 \mathrm{~cm}$ (much smaller than local fluctuations in bed height) by sending an ultrasonic wave and measuring the time of reflection from the target. In Fig. 3 we

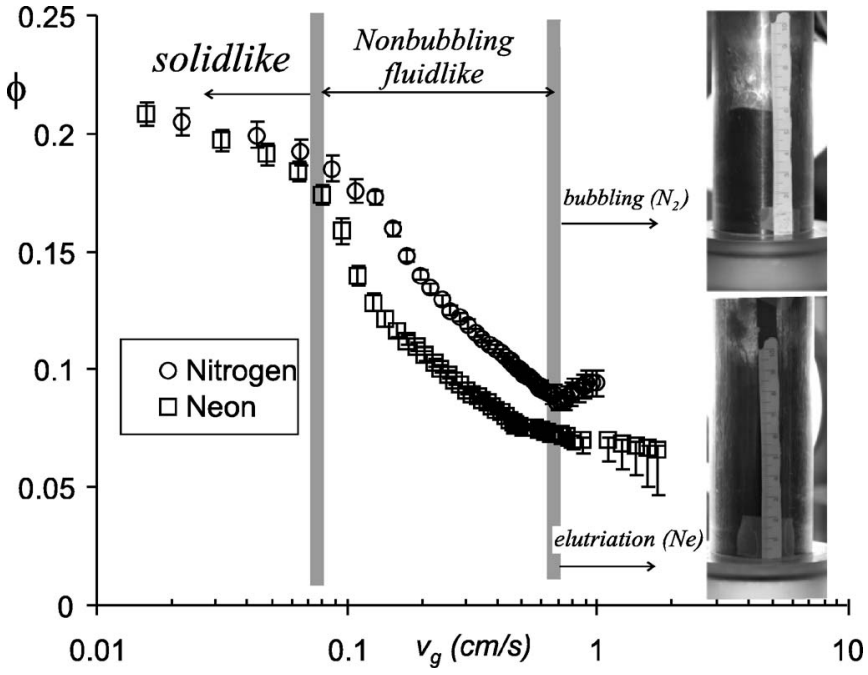

FIG. 3. Particle volume fraction measured vs gas velocity in fluidized beds of toner $\left(\rho_{p}=1135 \mathrm{~kg} / \mathrm{m}^{3}, d_{p}=7.8 \mu \mathrm{m}\right)$ with $\mathrm{N}_{2}$ and $\mathrm{Ne}$ at ambient conditions. Fluidization regimes are delineated. Inset: photographs of the fluidized bed in the bubbling regime (top) and elutriation regime (bottom).

plot experimental data of $\phi$ versus gas velocity $v_{g}$ for fluidization with both gases. It is seen that the type of gas does not play a major role on bed expansion in the solidlike regime as we would expect since the hydrodynamic interaction is not relevant. On the other hand the bed expands to smaller values of $\phi$ for fluidization with neon in the fluidlike regime. In agreement with our prediction the bed fluidized with nitrogen transits to bubbling and the one fluidized with neon transits to elutriation. In the former case $\phi$ reaches a minimum at the bubbling transition, characterized by oscillations of the bed height due to bubbles burst on the free surface, and the elutriated mass of powder is small. In the latter the height of the diffused free surface and the mass of elutriated powder increase monotonically as the gas velocity is increased.

Our diagram can be used to predict the fluidization behavior of powders as affected by other parameters such as interparticle force, fluid density, effective acceleration, and fractal dimension of the aggregates. Enhancing $F_{0}$ would shift the behavior from SFE to SFB to $A$ and from $B$ to $A$. A number of workers have shown how enhancing $F_{0}$ can shift the behavior from Geldart $B$ to $A$. For example, Geldart $B$ fluidization behavior gradually changed to $A$ by incremental addition of a liquid into the bed, by imposing a magnetic field on a bed of iron particles, or by using highly adsorbing gases [27]. Geldart $A$ behavior is predicted to shift to $B$ in a centrifugal field in accordance with experimental findings [28]. On the other hand, operating the fluidized bed in microgravity should lead to a full suppression of bubbling according to our diagram.

In conclusion, a predictive diagram of the type of gas fluidization expected for preconditioned fine cohesive powders has been proposed. Traditionally gas-fluidized beds and liquid-fluidized beds had been considered as disparate systems showing well-differentiated behaviors. In our approach we have extended empirical criteria mostly used in the past to explain the behavior of liquid-fluidized beds, showing in- 
stead that both systems can be understood from a general frame. Our approach to treat gas-fluidized beds of fine cohesive particles considers aggregates, grown to a size limited by the balance between interparticle force and flow shear, as effective low-density particles. In accordance with experimental observations our diagram predicts that the nonbubbling fluidization regime can be just solidlike for slightly cohesive particles. When particle size is decreased we predict the existence of a nonbubbling fluidlike regime as recently observed experimentally. For sufficiently small particles and/or high-viscosity gases, the fluidized bed transits directly from the nonbubbling fluidlike regime to elutriation as observed in gas-fluidized beds of nanoparticles, fluidized beds of micrometric particles with high-viscosity gas, and liquid-fluidized beds of moderate density large beads.

We acknowledge Spanish Government Agency Ministerio de Ciencia y Tecnologia (Contract No. FIS/2006-03645) and Junta de Andalucia (Contract No. FQM 421).
[1] R. Jackson, The Dynamics of Fluidized Particles (Cambridge University Press, Cambridge, U.K., 2000).

[2] S. Sundaresan, Annu. Rev. Fluid Mech. 35, 63 (2003).

[3] A. Castellanos, Adv. Phys. 54, 263 (2005).

[4] K. Rietema, The Dynamics of Fine Powders (Elsevier, London, 1991).

[5] D. Geldart, Powder Technol. 7, 285 (1973).

[6] J. M. Valverde, A. Castellanos, and M. A. S. Quintanilla, Phys. Rev. Lett. 86, 3020 (2001).

[7] R. Pfeffer et al., U.S. Patent No. 2006086834 (2006).

[8] C. Zhu, et al., AIChE J. 51, 426 (2005).

[9] J. M. Valverde and A. Castellanos, Phys. Rev. E 74, 021302 (2006).

[10] J. M. Valverde, M. A. S. Quintanilla, A. Castellanos, and P. Mills, Phys. Rev. E 67, 016303 (2003).

[11] D. Harrison et al., Trans. Inst. Chem. Eng. 39, 202 (1961).

[12] G. K. Batchelor, J. Fluid Mech. 193, 75 (1988).

[13] G. K. Batchelor, J. Fluid Mech. 119, 379 (1982); G. K. Batchelor and C.-S. Wen, ibid. 124, 495 (1982).

[14] E. Guazzelli, The Physics of Granular Media (Wiley-VCH, Berlin, 2004), p. 213.

[15] R. Zenit et al., J. Fluid Mech. 353, 261 (1997).
[16] S. Rapagna, P. U. Foscolo, and L. G. Gibilaro, Int. J. Multiphase Flow 20, 305 (1994).

[17] J. M. Valverde et al., Europhys. Lett. 54, 329 (2001).

[18] J. M. Valverde, M. A. S. Quintanilla, and A. Castellanos, Phys. Rev. Lett. 92, 258303 (2004).

[19] O. Molerus, Powder Technol. 33, 81 (1982).

[20] M. J. Rhodes et al., Chem. Eng. Sci. 56, 69 (2001).

[21] S. C. Tsinontides and R. Jackson, J. Fluid Mech. 255, 237 (1993).

[22] S. Ross and I. D. Morrison, Colloidal Systems and Interfaces (Wiley-Interscience, New York, 1988).

[23] J. M. Valverde and A. Castellanos, AIChE J. 52, 838 (2006); C. H. Nam et al., ibid. 50, 1776 (2004).

[24] X. S. Wang et al., Chem. Eng. Sci. 61, 8040 (2006).

[25] L. F. Hakim et al., Powder Technol. 160, 149 (2005).

[26] J. M. Valverde, A. Castellanos, and M. A. S. Quintanilla, Contemp. Phys. 44, 389 (2003).

[27] J. P. K. Seville and R. C. Clift, Powder Technol. 37, 117 (1984); H.-Y. Xie and D. Geldart, ibid. 82, 269 (1995); S. C. Saxena and W. Y. Wu, Can. J. Chem. Eng. 77, 312 (1999).

[28] G.-H. Qian, et al., AIChE J. 47, 1022 (2001). 